\title{
Where Are Adolescents with Cutaneous Melanoma Treated? An Italian Nationwide Study on Referrals Based on Hospital Discharge Records
}

\author{
Andrea Ferrari ${ }^{1}$, Alice Bernasconi ${ }^{1}$, Giovanna Sironi ${ }^{1}$, Laura Botta ${ }^{1}$, Stefano Chiaravalli ${ }^{1}$, \\ Michela Casanova ${ }^{1}$, Luca Bergamaschi ${ }^{1}$, Patrizia Gasparini ${ }^{1}$, Claudio Spinelli ${ }^{2}$, and \\ Annalisa Trama ${ }^{1}$ \\ ${ }^{1}$ Fondazione IRCCS Istituto Nazionale dei Tumori \\ ${ }^{2}$ University of Pisa
}

May 26, 2020

\begin{abstract}
We analyzed the nationwide hospital discharge records of adolescent inpatients in Italy to map where adolescents with melanoma are treated and identify expert centers. We identified 137 hospitals pointing out a dispersion of these patients that must be addressed to ensure to all adolescents with melanoma adequate treatment and enrolment in clinical studies.
\end{abstract}

\section{INTRODUCTION}

Cutaneous melanoma is a very common malignancy in adults in Australia, USA, Canada and Europe with a crude incidence rate of 71, 27, 26 and 23 per 100,000/year, respectively (https://gco.iarc.fr/).

The risk of developing melanoma increases with age and is therefore considered rare in the pediatric world. However, while it is very rarely seen in children under 10 years old (annual incidence around 0.7-0.8 per million), melanoma diagnoses definitely increase after puberty, with an estimated incidence of 14 and 23 per million in adolescent males and females ${ }^{1}$, respectively.

According to the consensus promoted by the European Cooperative Study Group for Pediatric Rare

Tumors (EXPeRT) and the European Union Joint Action on Rare Cancers (JARC), "very rare pediatric cancers" are those with an annual incidence $<2$ per million. ${ }^{2}$ This means that melanoma must be considered rare in children, but this may not be true in adolescents.

Melanoma in young patients is clinically challenging because it might have clinical features different from those usually seen in adults (for example, it might appear amelanotic or raised) ${ }^{3-5}$ and it is pathologically challenging since it may be difficult to differentiate truly malignant lesion from atypical tumors with uncertain malignancy. 6,7

Furthermore, the understanding of the disease in adolescents is limited because clinical studies on melanoma do not generally include young patients. This is of particular concern considering the huge change in the landscape of treatments for adult melanoma. ${ }^{8-11}$ In fact, the same therapeutic improvements have not been observed unfortunately in children and adolescents with melanoma, who still remain an inadequately served population. ${ }^{12}$

Against this background, we analyzed the hospital discharge records (HDRs) of adolescent inpatients newly diagnosed with cutaneous melanoma in the years 2007-2014 in Italy to identify where adolescents with 
melanoma are treated. Our idea is that mapping the hospitals where adolescents with melanoma are treated can contribute to identify expert centers and therefore promote collaboration for both, clinical management and clinical studies development.

\section{METHODS}

The methodology is described elsewhere (Ferrari et al $2019^{13}$ ). Briefly, we included the HDRs nationwide of all adolescents hospitalized in the years 2002-2015. Among them, we selected cases with at least one hospital stay for a diagnosis of cutaneous melanoma (ICD-9-CM diagnostic code $=172 *$ "Malignant melanoma of skin" and/or ICD-9-CM diagnostic code = V1082 "Personal history of malignant melanoma of skin") found in all the six diagnostic fields (main diagnosis and up to 5 secondary diagnosis) of the HDR. The first hospitalization indicating a diagnosis of cutaneous melanoma was defined as the index hospitalization. To select only incident cases, we excluded patients who were hospitalized during the 5 years or more before their index hospitalization (i.e. prevalent cases) for the same diagnosis or for diagnosis referring to cancer or its treatment. The diagnostic procedures and main treatments for melanoma were established searching in all the six procedural fields (main procedure and up to 5 secondary procedures) melanoma-specific ICD9-CM procedure codes: melanoma surgical interventions were divided between radical excision (ICD-9-CM procedure code $=86.4$ "Radical excision of skin lesion") and local excision (ICD-9-CM procedure code=86.3 "Other local excision or destruction of lesion or tissue of skin and subcutaneous"). We defined as main treatments those begun within 12 months of the diagnosis. Thus, study cohort starts in 2007 rather than 2002 and ends in 2014 rather than 2015. The treatment of each adolescent with melanoma was associated with only one hospital. When patients were treated at different hospitals, we assigned them to the hospital performing the radical excision or, if a radical excision was not performed, we assigned them to the hospital performing the local excision. Patients without any hospitalization for neither radical or local excision were assigned to the hospital where they were diagnosed (this applied to $12 \%$ of the adolescents with melanoma). The hospitals were divided into those having a unit affiliated to the national pediatric oncology network Associazione Italiana Ematologia Oncologia Pediatrica (AIEOP) - Italian Association of Pediatric Hematology and Oncology - and those without it (non-AIEOP hospitals).

Ethical approval for this study was obtained from the Ethical Commitee of Fondazione IRCCS Istituto Nazionale dei Tumori (N. INT 132/17).

\section{RESULTS}

We identified 418 adolescents (231 females and 187 males) newly diagnosed with cutaneous melanoma in Italy over a period of 8 years (from 2007 to 2014). These patients were referred to 137 different hospitals. Overall, 135 patients (32\% of the entire sample) were seen in 38 AIEOP- hospitals while 283 (68\%) in 99 non-AIEOP hospitals. Geographical dispersion of AIEOP (red dots) and non-AIEOP (black dots) hospitals across the country is represented in Figure 1.

\section{DISCUSSION}

These results point out a dispersion of the adolescent with melanoma cases in several hospitals and across different type of expertise, in fact we noticed that adolescents with melanoma were treated in different type of unit: pediatric and adult oncology, adult general surgery and dermatology.

Our results confirm a previous Italian analysis showing that only one in three children and one in ten adolescents with melanoma were treated within an Italian pediatric oncology center ${ }^{14}$.

This is of great concern considering the rarity of the disease in these young patients and call for an urgent rethinking of the way these patients are managed and, more important, of how clinical study should be organized.

Close cooperation between pediatric oncologists/surgeons and experts at specialist adult melanoma centers has been recommended. ${ }^{15}$ In particular, cooperative networking is necessary for clinical studies. ${ }^{16}$ Two 
international early-phase trials on new agents (involving vemurafenib and ipilimumab, respectively) were both prematurely closed due to insufficient recruitment of young melanoma patients. ${ }^{17,18}$

Our data should warn those who want to plan a cooperative study on melanoma in adolescents, to involve both pediatric oncology centers and adult centres or, include adolescents with melanoma in phase I/II clinical trials dedicated to adults considering that most drugs may have similar pharmacokinetics, tolerability profiles, and recommended doses for adolescents relative to adults ${ }^{19}$. The latter approach has been supported, among the others, by the ACCELERATE project promoted by the European Society for Paediatric Oncology (SIOPE) and the European Innovative Therapies for Children with Cancer (ITCC) Consortium (https://www.accelerate-platform.org/).

\section{CONFLICT OF INTEREST}

All the authors have no affiliation with any organization with a direct or indirect financial interest in the subject matter discussed in the manuscript.

\section{ACKNOWLEDGMENTS}

This work was supported by 5 x 1000 Funds - 2013, Italian Ministry of Health.

\section{DATA AVAILABILITY}

Data sharing is not applicable to this article. Authors do not have the permission to share data.

\section{REFERENCES}

1. AIRTUM, Italian Cancer Figures - Report 2012 Cancer in children and adolescents Epidemiol Prev 2013; 37(1 Suppl 1): 1-296

2. Ferrari A, Brecht IB, Gatta G, et al. Defining and listing very rare cancers of pediatric age: consensus of the Joint Action on Rare Cancers (JARC) in cooperation with the European Cooperative Study Group for Pediatric Rare Tumors (EXPeRT). Eur J Cancer . 2019; 110: 120-126.

3. Cordoro KM, Gupta D, Frieden IJ, McCalmont T, Kashani-Sabet M. Pediatric melanoma: results of a large cohort study and proposal for modified ABCD detection criteria for children. J Am Acad Dermatol. 2013; 68: 912-925.

4. Ferrari A, Bono A, Baldi M, et al. Does melanoma behave differently in younger children than in adults? A retrospective study of 33 cases of childhood melanoma. Pediatrics. 2005; 115 (3): 649-54

5. Han D, Zager JS, Han G, et al. The unique clinical characteristics of melanoma diagnosed in children. Ann SurgOncol . 2012; 19: 3888-3895.

6. Spatz A, Ruiter D, Hardmeier T, et al. Melanoma in childhood: an EORTC-MCG multicenter study on the clinico-pathological aspects.Int J Cancer . 1996; 68: 317-324.

7. Massi D, Tomasini C, Senetta R, et al. Atypical Spitz tumors in patients younger than 18 years. $J$ AmAcad Dermatol. 2015; 72: 37-46.

8. Ascierto PA, McArthur GA, Dréno B, et al. Cobimetinib combined with vemurafenib in advanced BRAFV600-mutant melanoma (coBRIM): updated efficacy results from a randomised, double-blind, phase 3 trial.Lancet Oncol . 2016; 17:1248-1260.

9. Long GV, Stroyakovskiy D, Gogas H, et al. Dabrafenib and trametinib versus dabrafenib and placebo for Val600 BRAF-mutant melanoma: a multicentre, double-blind, phase 3 randomised controlled trial.Lancet . 2015; 386: 444-451.

10. Wolchok JD, Chiarion-Sileni V, Gonzalez R, et al. Overall survival with combined nivolumab and ipilimumab in advanced melanoma. $N$ Engl J Med . 2017; 377: 1345-1356.

11. Long GV, Hauschild A, Santinami M, et al. Adjuvant dabrafenib plus trametinib in stage III BRAFmutated melanoma. N Engl J Med . 2017; 377: 1813-1823.

12. Indini, Brecht IB, Del Vecchio M, Sultan I, Ferrari A. Cutaneous melanoma in adolescents and young adults. Pediatr Blood Cancer . 2018; 65(11): e27292.

13. Ferrari A, Bernasconi A, Sironi G, et al. Where Are Adolescents with Soft Tissue Sarcomas Treated? An Italian Nationwide Study on Referrals Based on Hospital Discharge Records. J Adolesc Young Adult 
Oncol . 2020; 9 (2): 190-195

14. Ferrari A, Bisogno G, Cecchetto G, et al. Cutaneous melanoma in children and adolescents: the Italian Rare Tumors in Pediatric Age Project Experience. J Pediatr . 2014; 164: 375-382

15. Wouters MW, Michielin O, Bastiaannet E, et al. ECCO essential requirements for quality cancer care: melanoma. Crit Rev Oncol Hematol . 2018; 122: 164-178.

16. Ascierto PA, McArthur GA, Dréno B, et al. Cobimetinib combined with vemurafenib in advanced BRAFV600-mutant melanoma (coBRIM): updated efficacy results from a randomised, double-blind, phase 3 trial.Lancet Oncol . 2016; 17: 1248-1260.

17. Chisholm JC, Suvada J, Dunkel IJ, et al. BRIM-P: a phase I, open-label, multicenter, dose-escalation study of vemurafenib in pediatric patients with surgically incurable, BRAF mutation-positive melanoma. Pediatr Blood Cancer . 2018; 65: e26947.

18. Geoerger B, Bergeron C, Gore L, et al. Phase II study of ipilimumab in adolescentswith unresectable stage III or IV malignant melanoma.Eur J Cancer . 2017; 86: 358-363.

19. Momper JD, Mulugeta Y, Green DJ, et al. Adolescent dosing and labeling since the Food andDrug Administration Amendments Act of 2007.JAMA Pediatr. 2007; 167: 926-932.

\section{FIGURE LEGENDS}

Figure 1. Map of AIEOP (red dots) and non-AIEOP (black dots) centers treating adolescents with melanoma in Italy 


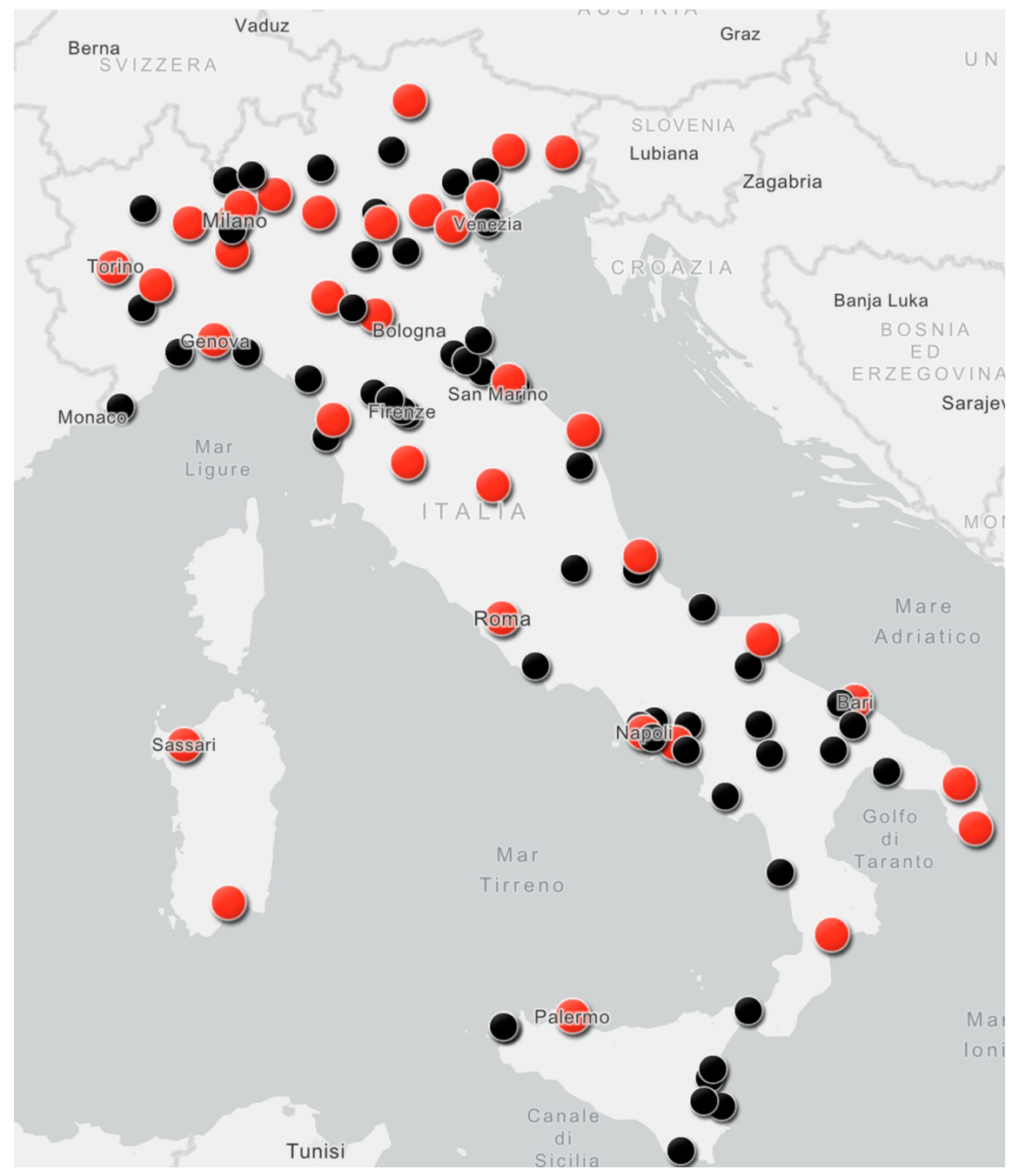

